\title{
RECHERCHES SUR LES PIROPLASMOSES DU PORC
}

\author{
Par Carlo G. CERRUTI (1)
}

La piroplasmose porcine est une maladie observée assez rarement et imparfaitement connue dans ses divers aspects épidémiologique, clinique, anatomo-pathologique et parasitologique ; il est probable cependant qu'elle est plus fréquente qu'on ne le croit communément, à en juger par le nombre de cas signalés dans ces dernières années, spéciaiement en Italie. C'est pourquoi je pense utiie d'exposer ici ce que l'on sait actuellement de cette maladie et les observations personnelles que j'ai eu l'occasion de faire en Sardaigne depuis 1934 .

Historique. - La maladie a été signalée pour la première fois en Russie en 1911 par Kowalewski qui, avec Dementieff, dit avoir observé chez des porcs, présentant de la gastro-entérite avec fièvre, inappétence, amaigrissement et anémie, des altérations sanguines (anisocytose et poikilocytose) avec des inclusions spéciales dans les hématies; ces formations arrondies ou annulaires, isolées ou bigéminées, se coloraient en bleu par le bleu de méthylène et disparaissaient du sang après traitement par la quinine et l'atoxyl ; pour cela, elles furent considérées comme de nature parasitaire et rapportées à un piroplasme. On se demande pourtant actuellement si Kowalewski et Dementieff eurent bien affaire à une hémosporidie ; en fait, Knuth et Du Toit trouvent étrange que le noyau qui se colore en pourpre par le Giemsa ait pu échapper aux observateurs russes.

Peu après, en 1914, dans un rapport au Gouverneur de l'Afrique Orientale allemande, Trautmann signala l'existence de la maladie dans cette colonie, mais il ne publia pas ses observations, qui ne furent connues que plus tard, en 1921, gràce à Knuth et Du Toit. Ces auteurs nommèrent le parasite Piroplasma trautmanni et notèrent ses affinités morphologiques avec $P$. bigeminum, $P$. caballi et $P$. canis : dans les préparations, en effet, il est rarement annulaire, mais se présente plutôt sous une forme amiboïde, ou en poire, ou en fuseau; les piroplasmes sont par paire dans l'hématie, séparés

(1) Travail traduit de l'italien par le Professeur G. Lavier, de Lille.

Annales de Parasitologie, T. XVII, $\mathrm{N}^{\circ} 2 .-1^{\text {er }} \operatorname{mars} 1939$, p. 114-136. 
ou réunis entre eux par l'extrémité effilée. Dans le parasite de Trautmann, ce qui est caractéristique, c'est l'appareil nucléaire qui présente un dimorphisme particulier ; on note un noyau grand, fréquemment d'aspect lâche, ou un noyau petit, rond, compact, déplacé vers l'extrémité arrondie, obtuse de l'organisme. La longueur du parasite oscille entre $2 \mu, 5$ et $4 \mu$ et la largeur entre $1 \mu, 5$ et $2 \mu$; la multiplication parait se faire normalement par gemmation. Les pores malades montraient de la faiblesse, de la dépression, de l'inappétence, une fièvre élevée $\left(40,41^{\circ}\right.$ C.), de la pâleur des muqueuses; l'anatomie pathologique révélait dans chaque cas des aspects d'anémie. Selon toute probabilité, le Piroplasma trautmanni, au moins en Afrique, serait transmis par Boophilus decoloratus.

Sparapani, en 1917, signala trois autres eas de piroplasmose porcine, diagnostiqués à l'abattoir communal de Venise ; il s'agissait de pores âgés de 8 à 11 moís, qui présentaient des muqueuses de teinte subictérique, une démarche chancelante et un amaigrissement considérable. A l'autopsie, il nota chez tous une forte splénomégalie, un foie augmenté, dégénéré et de coloration jaune, de la congestion rénale, des urines rouge foncé dans la vessie; des pétéchies et des hémorragies plus ou moins étendues sur l'épicarde, l'endocarde, la muqueuse vésicale, la muqueuse gastro-intestinale; une augmentation de volume des ganglions lymphatiques, etc. L'examen microseopique du sang circulant mit en évidence de nombreuses hématies présentant un parasite que, d'après sa structure, sa forme et ses dimensions, Sparapani n'hésita pas à identifier avec Piroplasma ovis, partant de cette consideration que quelques mois auparavant les pores en question avaient ingéré de la viande crue de moutons, morts probablement de piroplasmose ; l'existence de cette maladie fut, en effet, établie dans le troupeau après la constatation de la maladie des porcs.

Dans l'ètat actuel de nos connaissances, les déductions hasardeuses de Sparapani, en ce qui concerne le passage de l'infection des moutons aux pores par ingestion de viande infectée, ne peuvent être acceptées, et les expériences bien connues de Nawrotski vont à l'encontre de cette opinion. Il est au contraire plus logique d'admettre qu'il s'agit d'une apparition fortuite et contemporaine de l'infection dans les deux espèces animales et due à deux espèces différentes, malgré leur similitude morphologique.

En 1924, Lanzillo, en collaboration avec Fusco et Scandaliato, signala la maladie dans les marais de Grosseto; plus tard (1933), il en donna une étude plus détaillée et plus complète. D'après Lanzillo, la piroplasmose porcine entraine en Toscane de grands dom- 
mages dans l'élevage des races sélectionnées, et exceptionnellement seulement frappe les pores de la race locale, élevés à l'état libre et beaucoup plus rustiques ; l'apparition de la maladie se fait normalement au printemps et à l'automne, plus rarement aux autres saisons. Au point de vue clinique, Lanzillo a décrit une forme maligne, à évolution rapide, de type septicémique, avec fièvre élevée $\left(41-42^{\circ} \mathrm{C}\right.$.), dyspnée, anorexie, démarche incertaine et chancelante, muqueuses hypérémiées, urines hémoglobinuriques; et une forme bénigne à allure chronique, à type d'anémie presque toujours sans hémoglobinurie. Dans la forme maligne, on note des lésions de type septicémique; dans la bénigne, les caractéristiques communes aux anémies graves. Dans les globules rouges des pores malades, l'auteur a mis en évidence des parasites particuliers, semblables à Piroplasma bigeminum, mais de dimensions supérieures, habituellement en forme de poire, de fuseau, d'anneau, isolés ou réunis au nombre de 2, 3, 4 par hématie. Lanzillo a nommé ce parasite Piroplasma suis, sans penser qu'il présente, comme nous le verrons, de grandes analogies morphologiques avec $P$. trautmanni, l'espèce à laquelle il était le plus logique de la rapporter.

Cerruti, en 1934, a signalé le premier foyer de piroplasmose porcine en Sardaigne, et plus précisément dans la province de Cagliari, dû à un piroplasme de grande taille.

Abusalimoff, du laboratoire bactériologique de Bakou, a décrit et étudié très soigneusement en 1935 deux nouveaux foyers de piroplasmose porcine. Les animaux atteints présentaient une température élevée $\left(40-41^{\circ} \mathrm{C}\right.$.), les muqueuses ictériques et pàles, de la faiblesse, une démarche chancelante, de l'anorexie avec alternative de diarrhée et constipation. A l'autopsie, l'auteur nota un engorgement ganglionnaire, une teinte grisâtre du foie, un épanchement séro-hémorragique du péricarde, de petites hémorragies sous l'épicarde et sous la muqueuse gastrique, une coloration ictérique des séreuses et de la graisse. L'examen microscopique du sang mit en évidence la présence dans les hématies de parasites très semblables à ceux vus par Trautmann ; il s'agissait de piroplasmes d'aspect piriforme, parfois effilés aux deux extrémités, parfois de forme annulaire, longs de 3,33 à $6 \mu$ et larges de 1,67 à $2 \mu$, pourvus de chromatine réunie en un granule unique, parfois divisée en deux petites masses séparées, de grandeur différente, avec cytoplasme bleu-clair, seuls dans les hématies ou réunis au nombre de 2 à 4 et parfois même de 5 .

Tout récemment, Cavalletti a signalé l'existence de la piroplas- 
mose porcine en Ombrie, dans la commune de Castiglione del Lago (province de Pérouse). Les symptômes et les lésions notés dans ce foyer cö̈ncident avec ceux que nous avons déjà décrits; dans les hématies des porcs malades, Cavalletti a observé un parasite morphologiquement semblable à Piroplasma bigeminum.

A la fin du rapport de l'année 1937 sur l'activité de l'Istituto Zooprofilattico Sperimentale delle Tre Venezie, on apprend que dans le courant de cette année a été diagnostiqué un cas de piroplasmose porcine sans autre renseignement.

L'examen de ces données bibliographiques montre que le problème de la piroplasmose porcine, actuellement à peine ébauché, doit s'imposer à l'étude et attirer l'attention des chercheurs et des praticiens, parce que la maladie, qui a jusqu'ici échappé souvent à l'observation, cause des dommages économiques peut-être plus grands qu'on ne le croit communément.

\section{ObServations personnelles}

Pour la clarté d'exposition, j'étudierai dans des paragraphes distincts les divers aspects de la maladie, me rapportant surtout aux observations recueillies en Sardaigne pendant ces dernières années et qui sont valables aussi pour le continent italien.

Etude clinique. - Le tableau clinique de la piroplasmose porcine, décrit sommairement par les divers observateurs, est sensiblement uniforme et correspond à celui que j'ai observé.

Dans cette maladie, comme dans les autres formes des piroplasmoses, existent les quatre symptômes cardinaux, bien définis par Donatien et qui sont : fièvre, hémoglobinurie, ictère et anémie.

Nous ne pouvons rien dire sur la période d'incubation, qui ne pourra être établie que par l'observation prolongée et l'étude expérimentale ; tout laisse cependant supposer que cette période est semblable à celle qui a été déterminée pour les piroplasmoses des autres animaux domestiques : des semaines, peut-être même des mois.

La maladie débute avec des symptômes vagues, difficilement appréciables pour l'éleveur. On note d'abord uniquement de l'anc. rexie et de l'affaiblissement; les animaux perdent leur vivacité habituelle; ils s'écartent des autres et se nourrissent avec moins d'avidité qu'habituellement. A cet état de malaise général, s'ajoutent bientôt des phénomènes digestifs, caractérisés par des périodes de constipation, alternant avec une diarrhée plus ou moins violente, et semblables à celles qu'on observe dans les maladies infectieuses 
communes des pores et qui induiraient facilement en erreur si les autres symptômes n'apparaissaient pas bientôt. Le thermomètre révèle constamment une fièvre plus ou moins élevée et continue, avec de vastes oscillations s'élevant jusqu'à $39^{\circ} 5,40^{\circ}, 42^{\circ} \mathrm{C}$., accompagnée de dyspnée et de soif intense. Avant la mort, la température tombe brusquement à $37-38^{\circ} \mathrm{C}$. Les muqueuses visibles sont hypérémiées ; on peut y noter aussi quelques pétéchies ; les jours suivants apparaît progressivement une anémie profonde, tandis que la température oscille dans des limites assez vastes; les muqueuses se décongestionnent et se décolorent, preñant une teinte blanc-porcelaine qui, avec l'accentuation de l'anémie, devient subictérique ou franchement ictérique.

Dans les formes à allure plus lente, accompagnées de profonde destruction des globules rouges, et chez les animaux dont la peau n'est pas pigmentée, toute la superficie cutanée devient d'abord pâle, puis prend une teinte ictérique très caractéristique comparable à la couleur jaune de l'écorce du citron. La destruction des hématies est tellement énorme que l'on peut parler d'un véritable état hydrémique; en fait, la ponction d'un vaisseau superficiel laisse sortir un sang pâle et aqueux, avec un sérum de couleur jaune-citrin. Dans quelques cas graves, j'ai noté que le nombre des globules rouges pouvait tomber jusqu'à 1.200 .000 par mme. ; d'habitude, il se maintient à deux ou trois millions.

Les lésions sanguines sont toujours évidentes, constantes et caractéristiques, comme d'ailleurs on l'observe dans toutes les formes des piroplasmoses ; on note surtout de l'anisocytose, souvent très accentuée, qui commence avec l'accès aigu de la maladie et persiste encore quelques mois après la guérison; il y a aussi de la poikylocytose; de la polychromatophilie et de la basophilie; on observe aussi quelques hématies ponctuées avec granulations acidophiles, mises en évidence spécialement par la coloration de Stevenel-Zottner. A côté de ces altérations sanguines, on voit une quantitè notable de globules rouges nucléés (érythroblastes) et d'hématies contenant des corps de Jolly de forme régulièrement arrondie, à bords très nets, occupant dans l'hématie une position presque toujours centrale ; leur diamètre est de 1 à $2 \mu$ et leur teinte brunfoncé très intense.

La formule leucocytaire est très altérée ; on note en général une diminution du nombre des lymphocytes, des polynucléaires neutrophiles et éosinophiles, une disparition des basophiles et l'augmentation parfois considérable des grands mononucléaires et des myélocytes et méta-myélocytes (formes de transition) neutrophiles; la formule leucocytaire est donc modifiée dans le sens de la mono- 
cytose. La formule leucocytaire moyenne de la piroplasmose porcine pourrait s'établir ainsi :

Lymphocytes ........................ 14

Grands mononucléaires ................... 44

Myélocytes neutrophiles ................... 4

Métamyélocytes neutrophiles ................ 12

Polynucléaires neutrophiles $\ldots \ldots \ldots \ldots \ldots \ldots \ldots, 25$

- éosinophiles ................. 1

basophiles ................. 0

L'amaigrissement est très rapide et prononcé et va de pair avec l'anémie. Les urines sont constamment chargées d'hémoglobine, la couleur en varie de la teinte porto à celle de marc de café.

Au stade terminal de la maladie, les pores présentent toujours une démarche chancelante et des accès de grincements de dents, des aberrations du goût et des symptômes variés d'excitation nerveuse, caractérisés en particulier par la tendance à foncer furieusement contre l'homme et les autres animaux; la mort survient généralement dans le coma, comme cela se produit dans les piroplasmoses des autres animaux domestiques.

La durée de la maladie est habituellement de 7 à 8 jours; elle se termine presque constamment par la mort dans les cas oủ le traitement adéquat n'a pas été fait à temps.

A côté de cette forme clinique, que l'on pourrait appeler classique, dans quelques localités surtout des hauteurs, j'en ai observé une autre d'allure plus bénigne et caraclérisée par une symptomatologie plus discrète. Cette forme se manifeste presque exclusivement par une anémie plus ou moins profonde accompagnée d'une température oscillant entre 39,9 et $40^{\circ} \mathrm{C}$., avec muqueuses porcelaine ou sub-ictériques, phénomènes d'excitation nerveuse, troubles gastro-entéritiques plus ou moins évidents et graves. Les altérations du sang sont cependant notables et superposables à celles de la forme classique; le nombre des globules rouges oscille autour d'une moyenne de 4 millions par mmc. Dans cette forme bénigne aussi, la formule leucocytaire révèle une monocytose intense, accompagnée d'une diminution des polynucléaires neutrophiles:

Lymphocytes $\ldots \ldots \ldots \ldots \ldots \ldots \ldots \ldots \ldots \ldots \ldots, 22$

Grands mononucléaires .................. 36

Métamyélocytes (formes de transition) $\ldots \ldots \ldots \ldots, 7$

Polynucléaires neutrophiles $\ldots \ldots \ldots \ldots \ldots \ldots \ldots, 33$

- éosinophiles $\ldots \ldots \ldots \ldots \ldots \ldots, 1$

- basophiles ................... 1 
Les urines ne sont jamais hémoglobinuriques ; la mortalité est faible, presque négligeable; cette affection particulière, à allure lente, frappe tout particulièrement les porcelets de trois à cinq mois.

Anatomie pathologique. - Le tableau anatomo-pathologique est celui d'anémie grave et de cachexie. Les cadavres présentent une coloration ictérique intense de tous les organes, spécialement de la peau, du tissu cellulaire sous-cutané dans les régions dépourvues de pannicule adipeux, de la graisse, des séreuses, du cœur, des reins, du foie, des poumons et du cerveau ; le sang est aqueux avec sérum de couleur jaune-citrin; infiltration séreuse dans les masses musculaires qui sont plutôt flasques ; épanchement séreux citrin, parfois hémorragique dans les grandes séreuses et surtout le péricarde ; gangiions lymphatiques augmentés et succulents, parfois avec quelques points hémorragiques. La rate est toujours augmentée de volume, de consistance plutôt élastique, avec pulpe de couleur rose; le foie, hépatomégalique, peu succulent à la coupe, parfois fortement dégénéré ou d'aspect muscade, est friable; la vésicule biliaire est tendue et remplie d'une bile dense et trouble ; les reins présentent des signes de dégénérescence trouble; le myocarde a presque toujours l'aspect de viande bouillie, avec suffusions hémorragiques sous-épicardiques et sous-endocardiques, plus ou moins étendues et nombreuses, surtout visibles au niveau du sillon coronarien; les poumons sont quelquefois légèrement hypérémiés, mais d'habitude ne présentent pas de lésions ; la vessie, souvent distendue, contient de l'urine présentant les caractères décrits plus haut ; le tube digestif, parsemé de petites hémorragies punctiformes sous-muqueuses, a parfois un contenu hétérogène (sable, terre, etc.) ; la moelle osseuse est hémorragique dans les formes aiguës ou de consistance gélatineuse avec aspect jaunâtre dans les formes accompagnées d'anémie profondé ; dans la boîte cranienne, on ne note pas de lésions appréciables : il y a seulement congestion des vaisseaux méningés et une coloration jaunâtre de toute la masse encéphalique.

Histologie pathologique (1). - Les lésions histo-pathologiques relevées peuvent se résumer ainsi :

Foie. - Léger épaississement avec hyalinose à partir de la capsule de Glisson; le parenchyme du voisinage est quelque peu désor-

(1) J'ai le devoir de remercier vivement le professeur A. Busilcu, directeur de l'Institut d'Anatomie pathologique de l'Université R. de Cagliari, pour la courtoise hospitalité qu'il m'a donnée dans son Institut en vue de mes recherches histologiques et pour ses savants conseils dans l'interprétation des lésions. 
ganisé par la présence des faisceaux conjonctifs qui, de la capsule, plongent dans la profondeur : à côté d'îlots parenchymateux, plus grands, polygonaux, s'en trouvent d'autres plus petits, à contours irréguliers. Les traînées conjonctives sont formées par du conjonctif fibrillaire et présentent une quantité notable de cellules histioïdes et de lymphocytes. Les plus grandes de ces traînées se dessinent sur le parenchyme en rubans effilochés, mais en se limitant à des parties circonscrites des aires pseudo-acineuses.

Le parenchyme est profondément altéré ; il en reste, relativement bien conservés, de petits cordons disposés le long du périmètre des subdivisions pseudo-acineuses ; relativement bien conservés, en ce sens qu'on note à un plus fort grossissement, des cellules hépatiques fusionnées ou avec des contours mal définis, avec des vacuoles dans le protoplasme et parfois aussi dans le noyau.

Les capillaires intra-acineux sont dilatés; dans les autres parties de l'acinus, on note les limites mal définies des cellules hépatiques, en dégénérescence plus ou moins avancée ; çà et là, on voit une enclave verdâtre ou vert-jaunâtre, qui apparait formée par la bile; des granules de bile de dimensions variables se trouvent parfois disséminées dans le territoire parenchymateux en dégénérescence. Il y a des zones, au milieu du tissu parenchymateux profondément altéré, dans lesquelles les cellules hépatiques ont presque disparu et parmi les restes du stroma qui limite les espaces intercordonaux, on note un épanchement sanguin. Les globules rouges qui s'y trouvent prennent assez confusément la teinte de fond; on n'y voit aucune inclusion.

Les cellules de Kupfer, dans les zones de parenchyme encore relativement bien conservées, sont d'un volume un peu augmenté ; quelques-unes présentent de petites enclaves de pigments biliaires, différents des granules d'hématoxyline. La coloration de Giemsa permet de noter des éosinophiles, mais qui ne sont jamais très abondants et sont disséminés irrégulièrement.

Rate. - Le caractère le plus frappant des altérations atteignant cet organe est donné par une hyperplasie, aussi bien de la pulpe que des follicules. Les follicules montrent une augmentation numérique des éléments centro-folliculaires avec une réduction nette de l'auréole lymphocytaire. Dans le plan de section, ils apparaissent comme autant de petits foyers rapprochés, arrondis ou ovoïdes, avec réduction de calibre et disparition de l'artère pénicillaire ; ils tranchent comme des zones claires qui contrastent aussi dans la microphotographie; les seuls éléments constitutifs sont des cellules dont le protoplasme est très pâle, tlou, vacuolisé, 
Entre les follicules, il y a des ponts de tissu splénique dont les sinus sont comprimés par l'hyperplasie des cellules réticulaires et la présence de bandes de tissu conjonctif assez abondant. Dans la lumière de ces sinus, on note des cellules desquamées de la paroi et des cellules à noyau monocytoïde. On trouve aussi, avec une abondance variant suivant les champs, des granules de pigment brun, libres ou contenus dans le protoplasme des cellules foliacées. Les globules rouges apparaissent seulement en nombre restreint.

En plein parenchyme, on découvre une cavité sans aucun contenu, tapissée d'une mince membrane anhiste, d'aspect lamellaire, ayant la physiononie d'une cuticule parasitaire échinococcique. Cette cuticule repose sur une membrane de fibres collagènes et de cellules conjonctives. Dans un endroit du contour, entre la membrane conjonctive et la cuticule, il y a des formations polynucléées, constituées par beaucoup de noyaux pressés les uns contre les autres dans une zone protoplasmique homogène. Avec le Giemsa, on voit de rares cellules éosinophiles disséminées. La capsule splénique est légèrement épaissie.

Cœur. - Le cœur présente autour des vaisseaux des trainées d'épaississement conjonctif et des żones inégalement distribuées d'éclaircissement, avec atrophie des fibres musculaires et homogénéisation. Dans le myocarde se trouvent aussi de petits foyers ovoïdes formés d'une énorme masse de corpuscules arrondis ou allongés, contenus dans une mince membrane conjonctive.

Rein. - Dans les reins se trouvent épars de petits foyers de prolifération conjonctive et de sclérose, particulièrement dans la corticale; à leur niveau, les tubes urinifères sont rétrécis ou mème disparus ; ces foyers se trouvent aussi vers les glomérules, dont la capsule apparait alors distendue. Les tubes urinifères, particulièrement les tubuli contorti de premier et de deuxième ordre, présentent une certaine augmentation de volume des cellules de revêtement, qui produit une diminution et une déformation de la lumière. Dans beaucoup des cellules tubulaires et dans les histiocytes, dans les foyers de substitution du parenchyme, on trouve des granules de pigment brun-verdâtre, parfois en quantité notable. Les anses glomérulaires, si elles présentent parfois une certaine augmentation du noyau, ne montrent pas de modification notable, excepté la dilatation signalée.

Ganglions lymphatiques. - Les ganglions lymphatiques présentent les mêmes types d'altération que la rate, c'est-à-dire : hyperplasie des follicules, due aux cellules centro-folliculaires, hyperpla- 
sie des cellules histiocytaires des cordons, diminution de calibre des sinus et présence dans leur lumière de cellules desquamées, dont beaucoup contiennent des granules de pigment brun-doré. D'autres granules de même nature se trouvent libres parmi les cellules cordonales et dans le protoplasme des histiocytes, qui prolifèrent et sont très nombreux.

En résumé, les lésions histologiques sont superposables à celles que l'on voit dans les septicémies hémorragiques. Ce qui domine, ce sont les hémorragies et les foyers dégénératifs et nécroticohémorragiques; ceux-ci sont particulierement nets dans le foie qui, à ce point de vue, se montre le plus gravement atteint. Les signes de dégénérescence se rencontrent dans d'autres parenchymes, comme le rein, le cœur ; je crois que l'aspect de la rate et des ganglions lymphatiques mérite une mention spéciale : dans ces organes, en effet, à côté d'altérations régressives des éléments propres des follicules et de la pulpe rouge, on voit une hyperplasie notable des cellules réticulo-histiocytaires, qui s'orientent en forme de foyers granulomateux; enfin, les modifications régressives et nécrobiotiques, en correspondance avec ces foyers d'hyperplasie, sont fréquentes et étendues.

Dans la rate et dans le foie, les cellules réticulo-histiocytaires (cellules des sinus, cellules de Kupfer) présentent les signes d'une phagocytose active de restes globulaires ou de pigments hémoglobiniques, provenant de la destruction exercée par les parasites. La sidérose, autrement dit, est surtout endocellulaire dans ces éléments et est peu intense.

L'aspect de la rate et des ganglions lymphatiques est en harmonie avec la monocytose que montre l'examen direct du sang et est très vraisemblablement liée à une action à la fois destructrice pour les globules rouges et stimulante pour le tissu réticulo-histiocytaire.

La présence des éosinophiles n'est probablement pas attribuable à la maladie, car elle ne s'est pas montrée constante chez tous les animaux et elle n'apparaît pas dans la formule leucocytaire ; elle serait attribuable plutôt à une échinococcose. L'éosinophilie et la mononucléose ont été pourtant rencontrées aussi par d'autres observateurs (Velu). Il y a encore dans le tableau histologique une discrète participation du rein, et cela sous forme de foyers infiltratifs et de productions histiocytaires et conjonctives, corticales, spéciales : elles apparaissent comme des lésions sub-chroniques et semblent contemporaines des foyers conjonctifs qui intéressent le foie et évoluant parallèlement à eux. Ces manifestations d'hépatite interstitielle chronique se sont montrées importantes dans les cas chroniques (Carpano). 
Les altérations histologiques que nous venons de décrire correspondent en grande partie à celles notées par quelques observateurs modernes: Asdrubali, par exemple, décrivant la piroplasmose bovine dans la région de Pérouse, relève les altérations dégénératives des parenchymes et les aspects prolifératifs des éléments réticulo-endothéliaux du foie, de la rate et des ganglions; elles appuient les observations d'auteurs antérieurs (Jimenez de Asua, Dios, Zuccarini et Kuhn). L'étude plus poussée dans ce sens est due justement à ces observateurs argentins qui ont rapproché les lésions du système réticulo-endothélial, du système myéloïde et du lymphoïde. Le système réticulo-endothélial est en état d'hyper-activité; il présente des phénomènes prolifératifs, des changements de forme, des inclusions dans les cellules réticulaires et les macrophages (hémosidérose). En ce qui concerne le système myéloïde, l'érythropoïèse et la leucopoïèse sont presque inactives.

Comme ces auteurs, j'ai aussi observé sur les coupes des inclusions basophiles dans le protoplasme des macrophages et des cellules réticulo-histiocytaires; la méthode de Giemsa ne les différencie pas mieux; je crois moi aussi qu'il s'agit là d'inclusions chromatiques produites par la pycnose. Je n'ai pu mettre en évidence, malgré mes recherches prolongées, des aspects que l'on puisse rapporter nettement à des parasites; peut-être le prélèvement, la méthode de fixation en sont-ils responsables ; souvenonsnous d'ailleurs que l'on a aussi bien ce résultal négatif dans des organes de paludéens certains ; il est́ possible qu'après la mort de l'animal, le corps du parasite subisse des altérations importantes qui le masquent ou le rendent peu accessible aux méthodes ordinaires de coloration ; cette supposition est appuyée par le fait qu'on voit les parasites dans les frottis d'organes colorés par la méthode de May-Grünwald-Giemsa.

Agents étiologiques. - A l'examen des frottis de sang périphérique, de pulpe splénique et hépatique, de parenchyme rénal, de muscles cardiaques et de moelle osseuse, colorés par les méthodes de May-Grünwald Giemsa ou de Stévenel-Zottner, on note constamment la présence dans les hématies, et parfois aussi en dehors de celles-ci, de type divers de parasites présentant des caractéristiques morphologiques spéciales que je crois intéressant de décrire avec quelque détail.

Le premier type se présente comme un piroplasme de grande taille, qui peut prendre des aspects très divers : il y a des formes rondes ou annulaires, plus rarement ovales, formées d'un anneau de cytoplasme, qui entoure une vacuole peu colorée, et d'un noyau 
marginal ou central arrondi, dont la chromatine se colore plus ou moins intensément en rouge rubis. Parfois, la chromatine est disposée de façon plus dispersée en are sur une plus grande partie de l'anneau du cytoplasme; dans quelques exemplaires aussi, on note deux masses ou plus de chromatine, nettement séparées, ou réunies entre elles par de fins filaments de nature chromatique. Il existe aussi des éléments de forme plus ou moins nettement quadrangulaire, munis d'un appareil chromatique complexe. Le diamètre des formes rondes varie de 1,6 à $4 \mu$; les formes ovales mesurent en moyenne 2,3 à $3 \mu, 4$ de longueur sur 1,1 à $2 \mu, 4$ de largeur ; le côté des formes carrées mesure en moyenne $2 \mu, 5$ de longueur (fig. 1, 1-13).

A còté des précédents, on note en cutre des éléments piriformes ou en fuseaux, rarement d'aspect trapu (fig. 1, 17-18), à base large et extrémité opposée un peu émoussée; plus fréquemment, on en voit d'aspect très grêle et effilé, ayant une ou les deux extrémités très fines, orientés suivant l'équateur de l'hématie et constitués par du cytoplasme plus ou moins dense disposé à la périphérie en délimitant une vacuole centrale; la chromatine en est située de façon très variable et peut même être divisée en deux masses et plus, séparées plus ou moins nettement les unes des autres; les éléments .piriformes ou fusiformes mesurent de 2,1 à $6 \mu$ de longueur sur 0,8 à $2 \mu, 4$ de largeur (fig. 1, 14-20).

On rencontre aussi des parasites en division longitudinale ou même transversale (fig. 2, 21, 22, 32), d'assez nombreux éléments en division par gemmation (fig. 2, 25-29, 34, 35), et des formes amiboïdes; ces dernières se présentent comme de gros éléments d'aspect bizarre, pourvus d'un cytoplasme coloré en bleu plus ou moins intense et de chromatine réunie en un seul bloc ou divisée en plusieurs masses dont la situation est variable ; ces formes amiboïdes, toujours isolées dans le globule rouge, mesurent de 3 à $5 \mu, 5$ de longueur, sur 1,4 à $3 \mu$ de largeur (fig. 2, 23, 24, 29, 35).

J'ai rencontré enfin des éléments parasitaires d'aspect curieux qui rappellent les petites formes de Plasmodium falciparum, constituées par un granule plus ou moins volumineux de chromatine d'où partent de très minces petites bandes de cytoplasme, colorées très faiblement et dont le contour est très variable (fig. $2,38,39,40$ ).

On note également des parasites libres, en fuseaux effilés aux deux extrémités ou en poires, isolés ou réunis en couples qui mesurent de 3 à $4 \mu, 9$ de longueur sur 0,9 à 1,3 de largeur (fig. 2, 41-44).

Une hématie contient habituellement de 1 à 4 parasites, presque 


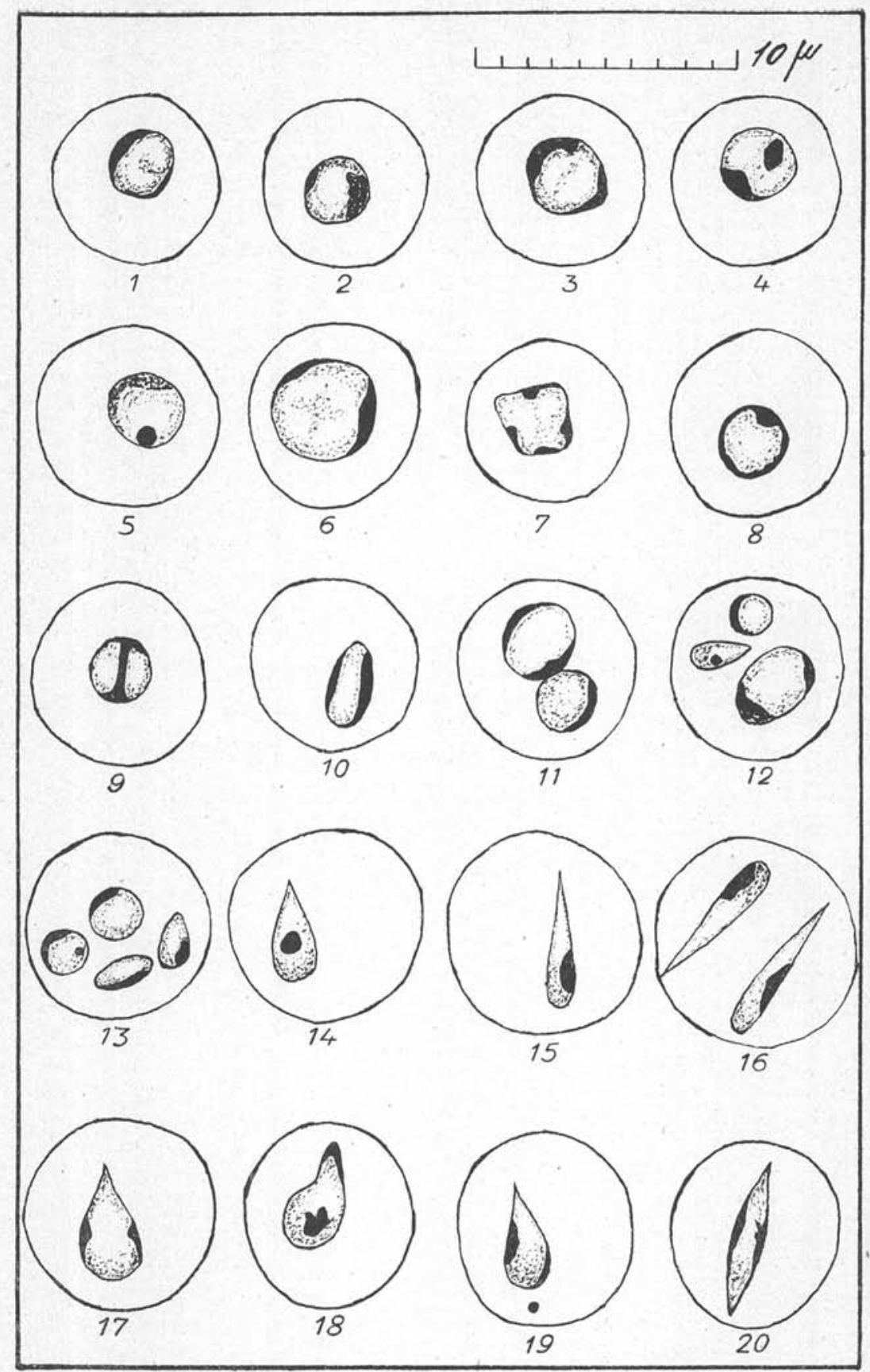

Fig. 1. - Pisoplasma trautmanni dans les frottis de sang périphérique. 
toujours de formes différentes et disposés de façon variable ; exceptionnellement, on peut en rencontrer 5 ou 6 (fig. 2,37 ); dans quelques frottis, les formes en marguerite ne sont pas rares (fig. 2, 36). Les formes en poire, presque toujours bigéminées, se disposent normalement suivant un angle aigu par rapport à leur axe longitudinal; plus rarement, elles sont réunies suivant un angle obtus ; parfois, un filament de cytoplasme réunit les extrémités effilées des deux parasites.

Le nombre des piroplasmes oscille entre des limites très grandes; dans quelques cas, ils sont extrêmement rares ; dans d'autres, par contre, ils parasitent de 60 à 65 p. 100 de la totalité des hématies. Le pourcentage des parasites par rapport aux hématies, quoique variable, est considérable ; dans quelques cas, j'ai pu compter 85 , 90 et même 120 parasites pour environ 100 globules rouges infestés ou normaux.

Le parasite que l'on rencontre en Sardaigne est morphologiquement identique à celui que l'on observe en Toscane et que j'ai eu la possibilité d'étudier sur des préparations que je dois à la courtoisie du $\mathrm{D}^{r}$ Lanzillo ; les microphotographies jointes sont très démonstratives à cet égard (pl. VII, fig. 1, 2, 3). Toutes deux correspondent d'ailleurs exactement aux descriptions et aux figures de Knuth et Du Toit, d'Abusalimoff et de Cavalletti. Les hémosporidies en question doivent donc être rapportées à l'espèce Piroplasma trautmanni Knuth et Du Toit, 1921 ; la dénomination Piroplasma suis Lanzillo, 1933, doit tomber en synonymie, d'après les règles internationales de la nomenclature zoologique.

Babesiellose porcine. - Dans les élevages porcins de la colonie pénale agricole de Castiadas (Cagliari), j’ai eı la possibilité, pendant les années 1935, 1937, 1938, d'étudier un parasite morphologiquement différent de Piroplasma trautmanni. Il se présente normalement dans les hématies (fig. 3 et 4 ) sous la forme d'un mince anneau de cytoplasme qui circonscrit une vacuole claire ; le cytoplasme contient une ou deux petites masses compactes de chromatine à rebords nets, qui tranchent très visiblement, situées à la périphérie du parasite, à la manière d'un chaton de bague simple ou double, ou plus ou moins enfoncées dans le cytoplasme. Le diamètre des formes annulaires mesure de 0,7 à $2 \mu$ (fig. $3,45-49$; fig. 4,71 ). Il $\mathrm{y}$ a aussi des formes ovalaires, quadrangulaires, lancéolées ou piriformes, qui reproduisent Ia même morphologie et qui mesurent de $1 \mu, 2$ à $2 \mu, 6$ de longueur sur $0 \mu, 7$ à $1 \mu, 9$ de largeur (fig. $3,50,52,57,58$; fig. 4, 72-87). On note 


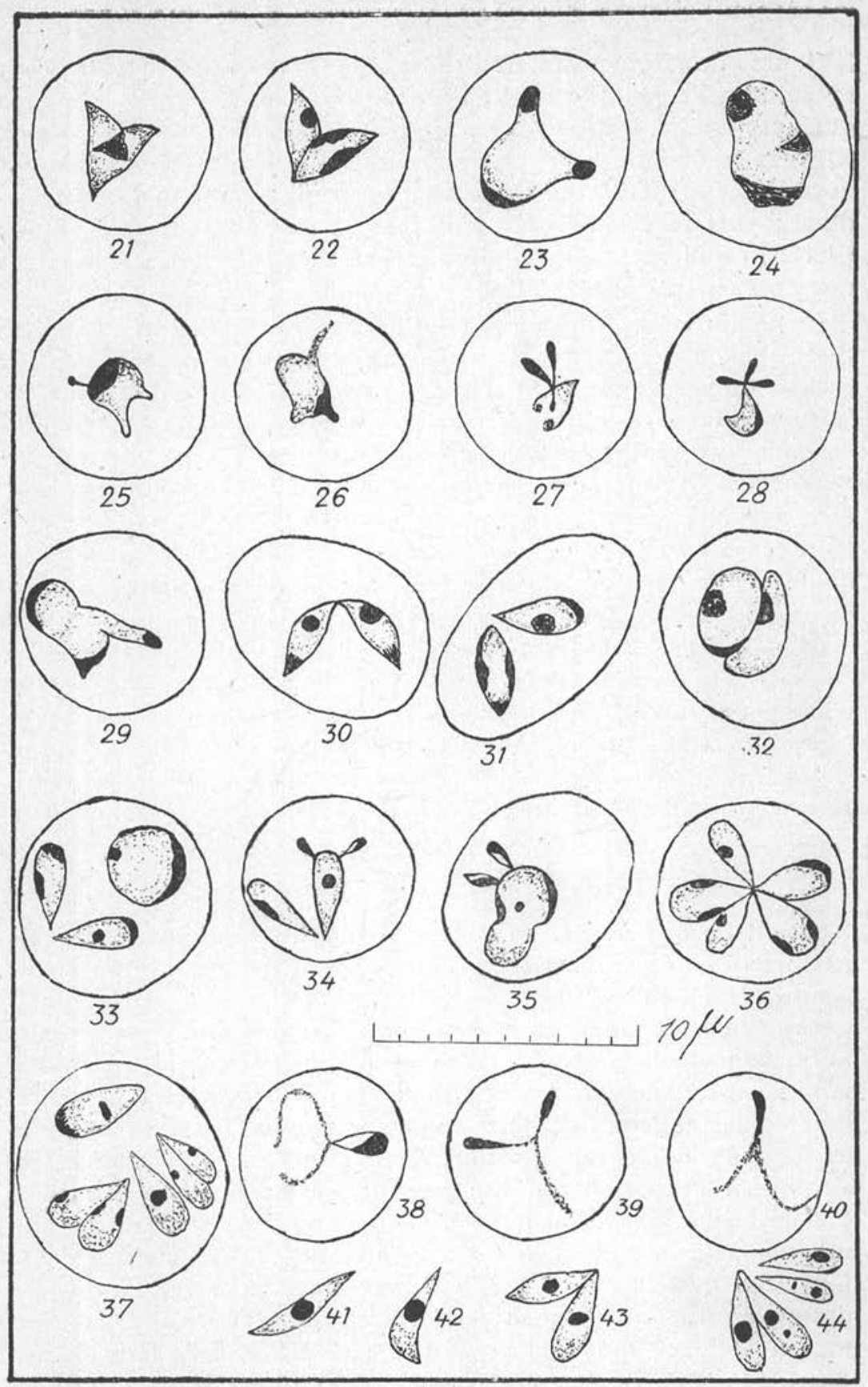

Fig, 2. - Piroplasma trautmanni dans les frottis de sang périphérique. 
enfin des éléments amiboïdes plutôt rares qui mesurent de 2,8 à $3 \mu, 2$ de longueur sur 1,8 à $2 \mu$ de largeur et de rares formes en division transversale, de dimensions plus grandes : 3,2 sur $1 \mu, 8$ (fig. $3,53,54,55,59)$. Les parasites endoglobulaires sont d'habitude isolés, mais on en observe un certain nombre assemblés par 2, 3, 4 dans les hématies, et même plus avec un nombre maximum de 8 . On note parfois des formes qui simulent la schizogonie. Le pourcentage des hématies parasitées est variable ; habituellement, on note un globule rouge infecté sur 10 à 15 .

Enfin, j'ai vu des formes libres très caractéristiques; dans un cas que j'ai observé en avril 1935, j'ai rencontré surtout dans la pulpe splénique et dans le muscle cardiaque des éléments de forme arrondie ou. légèrement ovale, semblables aux formes endoglobulaires déjà décrites, possédant comme elles une ou deux petites masses de chromatine à marges très nettes, isolés ou groupés par deux, trois ou quatre, et longs de 1,3 à $2 \mu, 3$ sur 1 à $1 \mu$, 3 . Ces éléments libres présentent quelque analogie morphologique avec les toxoplasmes (fig. 3, 65-69). Dans un cas observé l'année dernière, j'ai noté une prévalence de formes libres en poire, isolées ou accouplées, longues en moyenne de $1 \mu, 4$ sur 0,9 de largeur (fig. 4 , 90-91).

En ce qui concerne la forme des parasites, j'ai relevé la prévalence des éléments arrondis ou ovales sur les piriformes (70 contre 30 p. 100). Les parasites vus dans les cas observés en avril 1935 et en mai 1937 présentaient quelques légères différences morphologiques ; pour les premiers (voir fig. 3, 45-69) prévalaient les formes annulaires, tandis que chez les seconds (voir fig. 4, 70-87 et 90-91), on notait en majorité des éléments piriformes, formant avec leur axes longitudinaux un angle obtus, pouvant même atteindre $180^{\circ}$. Malgré ces légères différences de forme, ces piroplasmes me paraissent appartenir à une même espèce. Ces parasites du foyer de Castiadas, profondément différents de Piroplasma trautmanni, et jusqu'ici non encore signalés chez le porc, constituent à mon avis une espèce nouvelle du genre Babesiella Mesnil 1919 ; je propose done de leur donner, provisoirement au moins, le nom de Babesiella perroncitoi, en l'honneur de mon maître, l'illustre parasitologue Eduardo Perroncito, disparu en novembre 1936.

Corps anaplasmoïdes. - Dans les hématies du sang circulant des pourceaux atteints de la forme bénigne, caractérisée par une anémie plus ou moins profonde, j'ai constamment observé la présence de formations anaplasmoïdes particulières, presque toujours 


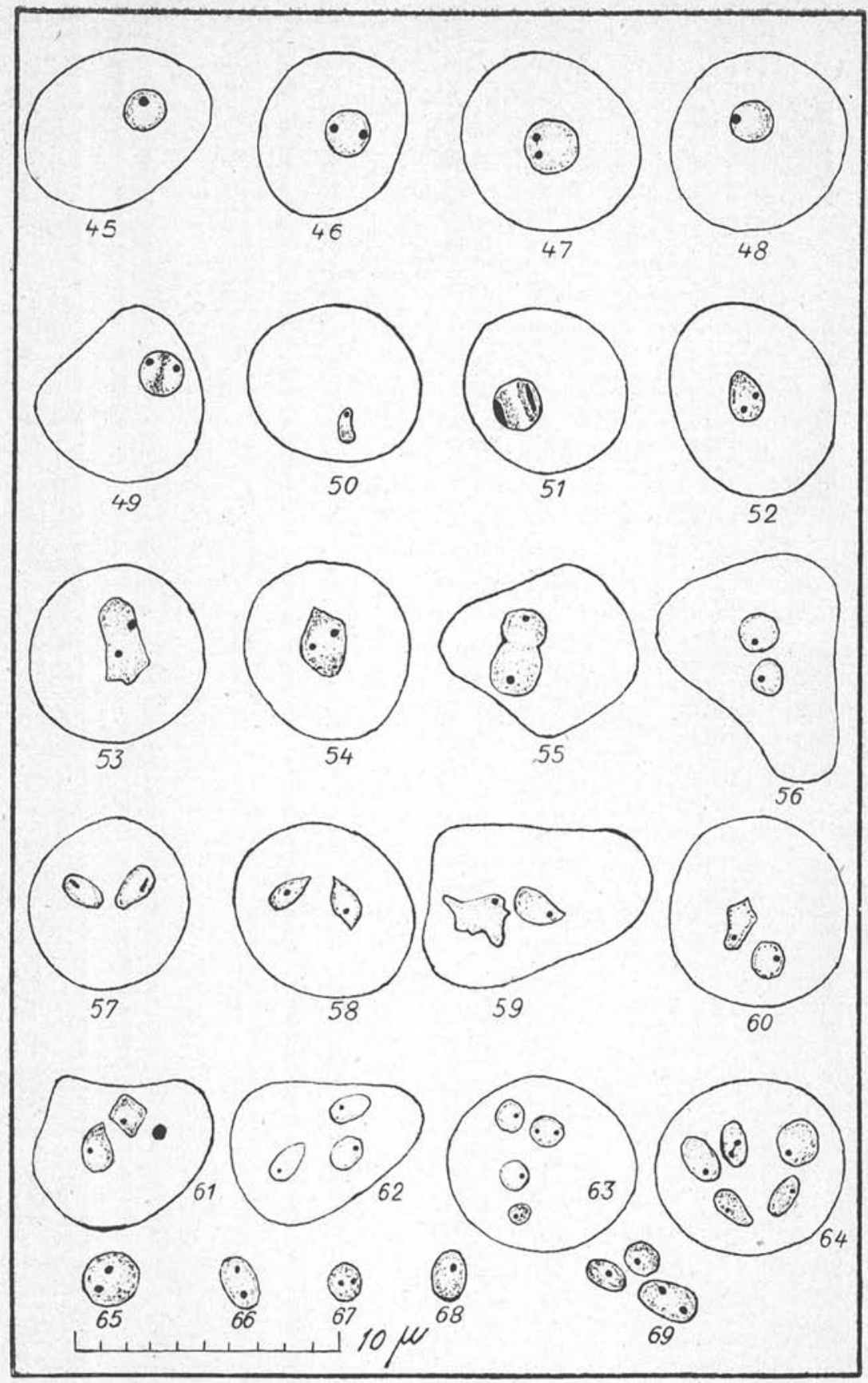

Fic. 3. - Babesiella perroncitoi; cas d'avril 1935. Frottis de sang périphérique et organes internes. 
situées en position marginale, à contours nets, à coloration rougeviolacé intense, dépourvues de cytoplasme et mesurant de 0,7 à $1 u$ de diamètre (fig. $4,88,89$ ). Ces formations particulières présentent de fortes analogies avec les anaplasmes vrais et aussi avec les corpuscules de Jolly desquels il n'est d'ailleurs pas possible de les différencier; aussi je ne puis me prononcer sur leur véritable nature.

La découverte de formes anaplasmoïdes dans les hématies des porcins de Sardaigne n'est pas nouvelle : Tibaldi, en 1914, dit avoir observé chez quelques porcs sur 150 examinés, des formes coccoïdes tantôt endocellulaires tantôt libres dans le plasma, prenant une teinte nettement chromatinique et sur la nature desquelles il ne s'est pas prononcé.

Dans l'état actuel de nos recherches, il serait très hasardeux d'affirmer que ces formations que l'on voit aussi dans le sang des porcs atteints de piroplasmose vraie ou de babésiellose sont ou non de nature parasitaire ; les expériences que je me propose de faire et surtout les inoculations, apporteront quelques éelaircissements sur leur signification biologique ; en tout cas, il est bien certain que l'on ne peut nier l'existence d'un lien évident entre ces corps anaplasmoïdes et l'anémie des porcelets.

Agents de transmission. - Sur la peau des porcs atteints de piroplasmose, j'ai capturé à diverses reprises et dans divers foyers quelques tiques des espèces suivantes: Rhipicephalus sanguineus Latreille 1804, Hyalomma xgyptium Linné 1758, Dermacentor reticulatus Fabricius 1794. Des recherches seront faites pour établir expérimentalement lesquels de ces arthropodes transmettent la maladie.

Epidémiologie. - Depuis ma première note de 1934, j’ai eu la possibilité d'étudier, dans la zone de plaines et de collines de la province de Cagliari, d'autres foyers de piroplasmose porcine identiques dans leurs manifestations cliniques et anatomo-pathologiques, bien que d'étiologie diverse.

On ne doit pas être très surpris par le fait qu'en Sardaigne, région où dominent assez gravement toutes les piroplasmoses des animaux domestiques, les cas parvenus à ma connaissance soient relativement peu nombreux. Normalement, dans cette région, comme dans le marais toscan, on élève des pores rustiques qui vivent à l'état sauvage ou demi-sauvage, qui ont toute possibilité de s'infecter quelques jours ou quelques semaines après la naissance et de contracter des affections inapparentes leur conférant 


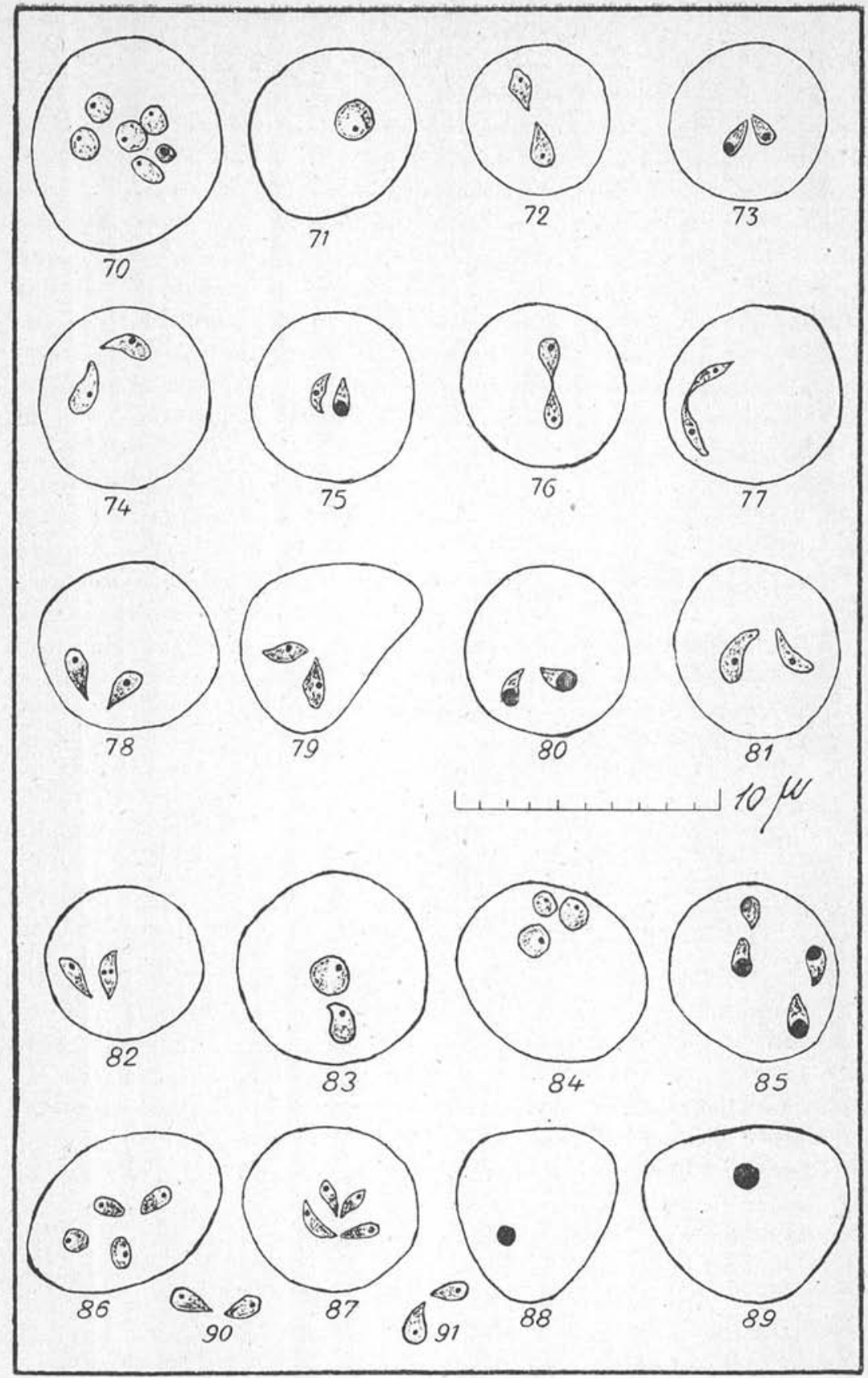

Fig. 4. - Babesiella perroncitoi ; fig. 70, cas d'avril 1935 ; fig. 71-87 et 90-91, cas de mai 1937 ; fig. 88-90, formes anaplasmoïdes de l'anémie des porcelets. Frottis de sang périphérique et d'organes internes. 
un état solide et durable de prémunition qui sera maintenu par de continuelles réinfections. Les pores, donc, en ce qui concerne leurs piroplasmoses, s'y comporteront comme les ovins sardes vis-à-vis de Babesiella ovis, Theileria recondita et Anaplasma ovis.

Le problème ne se pose pas de la même façon pour les pores de race sélectionnée, moins nombreux et élevés en porcheries, à l'abri des tiques, et qui ont ainsi moins de possibilité de contracter un état de prémunition dans le jeune âge ; c'est d'ailleurs dans ces élevages qu'il m'a été possible de faire mes premières observations ; enfin, des raisons locales évidentes, liées à la conscience hygiénique des éleveurs et sur lesquelles il est inutile d'insister gênent les enquêtes épidémiologiques sur la maladie.

En Toscane, d'après le $\mathrm{D}^{r}$ Lanzillo, la maladie produit des dommages considérables, dans cette partie de la Maremma constituant l'Agro grossettano, région de plaines et de marécages qui confine à la province de Livourne et s'étend le long du littoral thyrrénien jusqu'à Chiarone. Les cas observés par Lanzillo jusqu'en 1933, sont au nombre d'environ 6.000 ; dans une épizootie éclatée en mai-juin 1924 , le nombre des malades monta à 2.500 dont 598 moururent; les années suivantes on nota de graves épizooties, spécialement en 1926 et 1930 . Les premières observations de Cavalletti nous apprennent que la maladie ne constitue pas non plus une rareté en Ombrie, puisqu'au printemps de 1936 on observa 12 cas dans une seule commune (Castiglione del Lago); au printemps de l'année suivante, il y eut 36 cas, toujours dans cette même commune.

En Russie, d'après Abusalimoff, la maladie ne doit pas être considérée comme exceptionnelle et-si, dans la littérature russe on n'en trouve pas des mentions plus précises, cela signifie que les vétérinaires se sont jusqu'ici trop peu intéressés à cette question.

En Sardaigne, comme dans les autres régions, la maladie a une allure nettement saisonnière : habituellement, on la rencontre au printemps, rarement en été, souvent en automne ; des cas sporadiques peuvent aussi être vus pendant l'hiver et cela est lié à l'activité des tiques vectrices du parasite.

Thérapeutique. - La thérapeutique de la piroplasmose porcine, après les premières recherches de Dementiev qui a obtenu la disparition des parasites par administration de sels de quinine et d'atoxyl, a été étudiée plus récemment par Lanzillo et Cerruti.

Le premier emploie une préparation nommée "Apirosom », composée de bleu de méthylène, de bleu d'isamine et de jaune d'acridine en solution aqueuse qu'il injecte par voie sous-cutanée à la 
dose de 40 à $50 \mathrm{~cm}^{3}$. Les résultats seraient satisfaisants. J'ai eu de brillants résultats, même dans des cas très graves et à une période avancée, avec les injections d' «Acaprin 》 Bayer qui est doué d'un pouvoir chimiothérapique contre Piroplasma trautmanni; je préconise l'emploi de ce produit par voie sous-cutanée, à la dose de $15 \mathrm{cgr} .\left(3 \mathrm{~cm}^{3}\right)$ en renouvelant, quel que soit le résultat, la même dose 24 heures plus tard. Parmi les nombreux pores soignés par moi ou selon mes indications, un seul, qui présentait des symptômes d'hémoglobinurie intense, avec ictère, anémie profonde et infection massive des hématies, mourut 5 jours après l'injection de $5 \mathrm{~cm}^{3}$ d'Acaprin, et même pour cet animal le produit avait agi, puisque les urines redevinrent normales 24 heures après le traitement et que les frottis des organes exécutés après la mort furent microscopiquement négatifs. Cavalletti, sur mes indications, soumit au traitement à l'Acaprin 48 porcs, obtenant 46 guérisons et 2 insuccès imputables à l'intervention tardive, et même chez un de ces derniers, une heure après l'injection, les parasites disparurent du sang et des organes internes.

Egalement contre les infections par Babesiella perroncitoi, j'ai obtenu d'excellents résultats sur lesquels je me réserve de revenir dans une prochaine note.

\section{RÉsumÉ}

Les piroplasmoses porcines sont des maladies probablement plus répandues qu'on ne le croit actuellement. En Sardaigne, où les piroplasmoses des animaux domestiques sont communes, on en rencontre chez le porc.

Ces piroplasmoses porcines lèsent surtout les organes lymphopoiétiques et se traduisent par une grande destruction globulaire, entraînant une monocytose relative.

Elles sont dues à deux parasites différents : $1^{\circ}$ Piroplasma trautmanni Knuth et Du Toit 1921, qui produit la piroplasmose vraie, identique à celle de l'Italie continentale, de la Russie et de l'Afrique orientale ; $2^{\circ}$ Babesiella perroncitoi n. sp., qui produit la babésiellose porcine. Les deux affections sont d'ailleurs indistinguables du point de vue clinique et anatomo-pathologique.

Dans les hématies des porcelets atteints d'une anémie spéciale à allure bénigne, on trouve des corps anaplasmoïdes dont la signification biologique ne peut encore être précisée.

L' «Acaprin » Bayer possède un pouvoir thérapeutique spécifique contre Piroplasma trautmanni et Babesiella perroncitoi. 


\section{BiBLIOGRAPHIE}

Abusalmoff (M.). - Sowjetskaja Veterinarija, 1935, $\mathrm{n}^{\circ} 6$.

Asphubalı (M.). - La piroplasmosi dei bovini nella media valle del Tevere. Osservazioni sull'anaplasmosi e le sue lesioni. Rivista di biologia, XVIII, 1935 , fase. 1.

Barboni (E.). - L'anaplasmosi degli ovini dell' Umbria. Rivista di biologia, XIII, $1931, \mathrm{n}^{\circ \times} 1-111$.

Bardell1 (P.). - Relazione per l'anno 1937 dell' Istituto Zooprofilattico Sperimentale delle Tre Venezie, 1938, Tipografia del Seminario di Padova.

Bertolini (G.). - Piroplasmosi suina, in Igiene delle carni. Malattie del bestiame da macello, 1931, p. 458, U.T.E.T., Torino.

Carpano (M.). - La febbre della costa nella Colonia Eritrea. Clin. Veter., XXXV, 1912, p. 821.

Piroplasmosi tipo « parvum » nei bovini del basso bacino del Mediterraneo. Clin. Veter., XXXVIII, 1915, p. 497-553.

Cavalletti (S.). - Sulla piroplasmosi dei suini. Nuova Veter., XVI, 1938, p. 114.

Cenruti (C.-G.). - Ricerche sistematiche sugli emoprotozoi degli animali domestici della Sardegna. Atti del $V^{\circ}$ Congresso Nazionale di Microbiologia, Cagliari, 27-31 mai 1934, p. 55 .

Cerrruti (C.-G.) et Faxtoni (F.). - Ricerche sulla chemioterapia delle piroplasmosi con il nuovo prodotto Bayer Acaprin. Clin. Veter., LVIII, 1935 , p. 542.

Dementuew. - Zur Frage über Piroplasmose bei Schweinen. Bote $f$. allg. Veterinärwesen, $1911, \mathrm{n}^{\circ} 16$, p. 845.

Fusco (G.), Scandaliato (S.) et Lanzillo (V.). - Sulla piroplasmosi dei suini. Giornale di Medicina Veterinaria, LXXIII, 1924, p. 311.

Hutyra et Marek (J.). - Piroplasmi nei suini, in Patologia e Terapia speciale degli animali domestici, I, p. 1160; Vallardi, Milano, 1929.

Kikuth (W.). - Die Chemotherapie der Piroplasmosen. Zbl. f. Bakt., I., Orig., CXXV, 1935, p. 135.

Knuth (P.) et Du Tort (P.-J.). - III. Die Piroplasmose. 5. Die Piroplasmose des Schweines, in Messe, Handbuch der Tropen-Krankheiten, VI, 1921, p. 409 , Barth, Leipzig.

Kowalewski. - Sur la Piroplasmose du porc. Journal de Méd. Vét. et de Zootechn., 31 décembre 1911 , p. 714.

Jimenez de Asua (F.), Dios (R.-L.), Zúcarini (J.-A.) et KuhN (M.-J.). - Histopathologie de la «Tristeza \& (Piroplasmose et anaplasmose bovine). C.R. Soc. Biol., XCVII, 1927 , p. 1314.

Intervention du système réticulo-endothélial dans la \& Tristeza » (Piroplasmose et anaplasmose bovine). C.R. Soc. Biol., XCVII, 1927, p. 1341.

LaNzillo (V.). - La babesiosi o piroplasmosi dei suini nella Maremma Grossetana, 1933, Tipografia L'Elzevirana, Grosseto.

Nawrotzky (N.-N.). - Zur Piroplasmose-infektion der Hunde durch die Schleimhaut des Magen-Darmtraktes. Zbl. f. Bakt., I., Orig., LXVI, 1912 , p. 417.

Sengent (E.), Donatien (A.), Parrot (L.), Lestoguand (F.), Plantureux (E.) et Rougebief (H.). - Etudes expérimentales sur les piroplasmoses bovines d'Algérie. Ann. Inst. Pasteur, XXXVII, 1924, p. 273. 
Sparapani (C.-G.). - Trasmissione dell' infezione da Piroplasma ovis in tre suini per via digerente. Pathologica, IX, 1917, $\mathrm{n}^{\circ} 196$, p. 21.

Tibaldi (E.). - Forme di anaplasma nel sangue di diversi animali in Sardegna. Pathologica, VI, 1914, $\mathrm{n}^{\circ} 133$, p. 261.

Velu (H.). - Les piroplasmes et les piroplasmoses. Mémoires de la Société des Sc. nat. du Maroc, t. II.

\section{Station expérimentale zooprophylactique de Sardaigne} (Directeur : Professeur G. Pegreffi).

\section{EXPLICATION DES PLANCHES VII-IX}

\section{Planche VII}

Fig. 1 et 2. - Sang périphérique de porcins sardes infestés par Piroplasma trautmanni.

Fig. 3. - Sang périphérique d'un pore de la Maremma Grossettana (Toscane), infesté par Piroplasma trautmanni.

F1G. 4. - Sang périphérique de porcelet atteint d'anémie; on note deux corps anaplasmoïdes dans les hématies.

\section{Planche VIII}

Fig. 1. - Frottis de pulpe splénique de pore infesté par Babesiella perroncitoi (cas d'avril 1935).

FIG. 2. - Frottis de pulpe hépatique de porc infesté par B. perroncitoi (cas d'avril 1935).

FIG. 3. - Frottis de muscle cardiaque de porc infesté par B. perroncitoi (cas de mai 1937).

Fig. 4. - Frottis de pulpe hépatique de porc infesté par B. perroncitoi (cas de mai 1937).

\section{Planche IX}

En haut, coupe de foie de porc atteint de babésiellose; en bas, coupe de rate de porc atteint de babésiellose. 
ANNALES DE P.IRASITOLOGIE

T. XVII, No 2, 1 er Mars 1939
Planche VII

(Mémoire Cerreti)
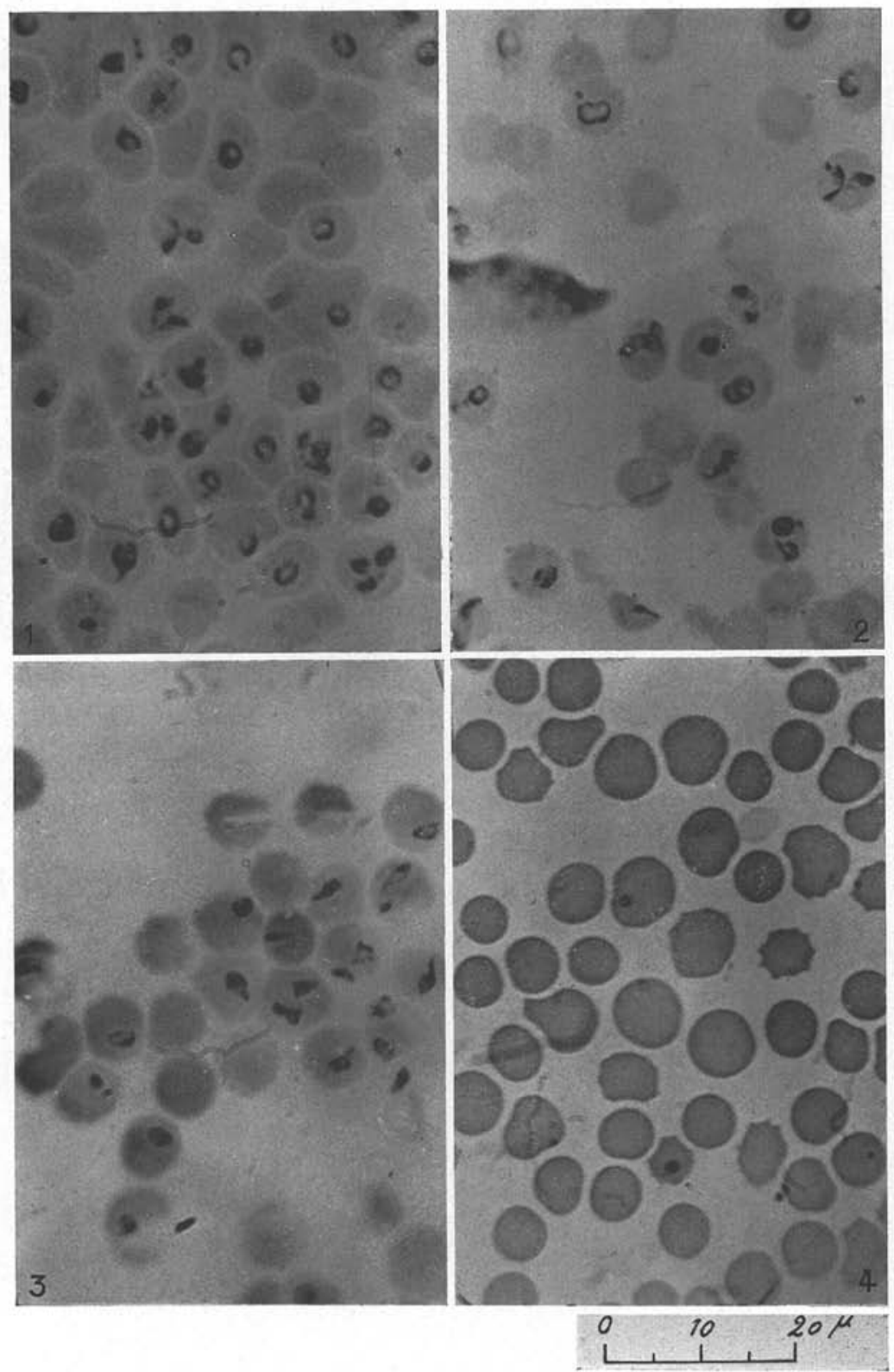

Masson Et Cie, Editeurs 
ANNALES DE PARASITOLOGIE

T. XVII, No 2, 1er Mars 1939
Planche VIII

(Mémoire Cerruti)
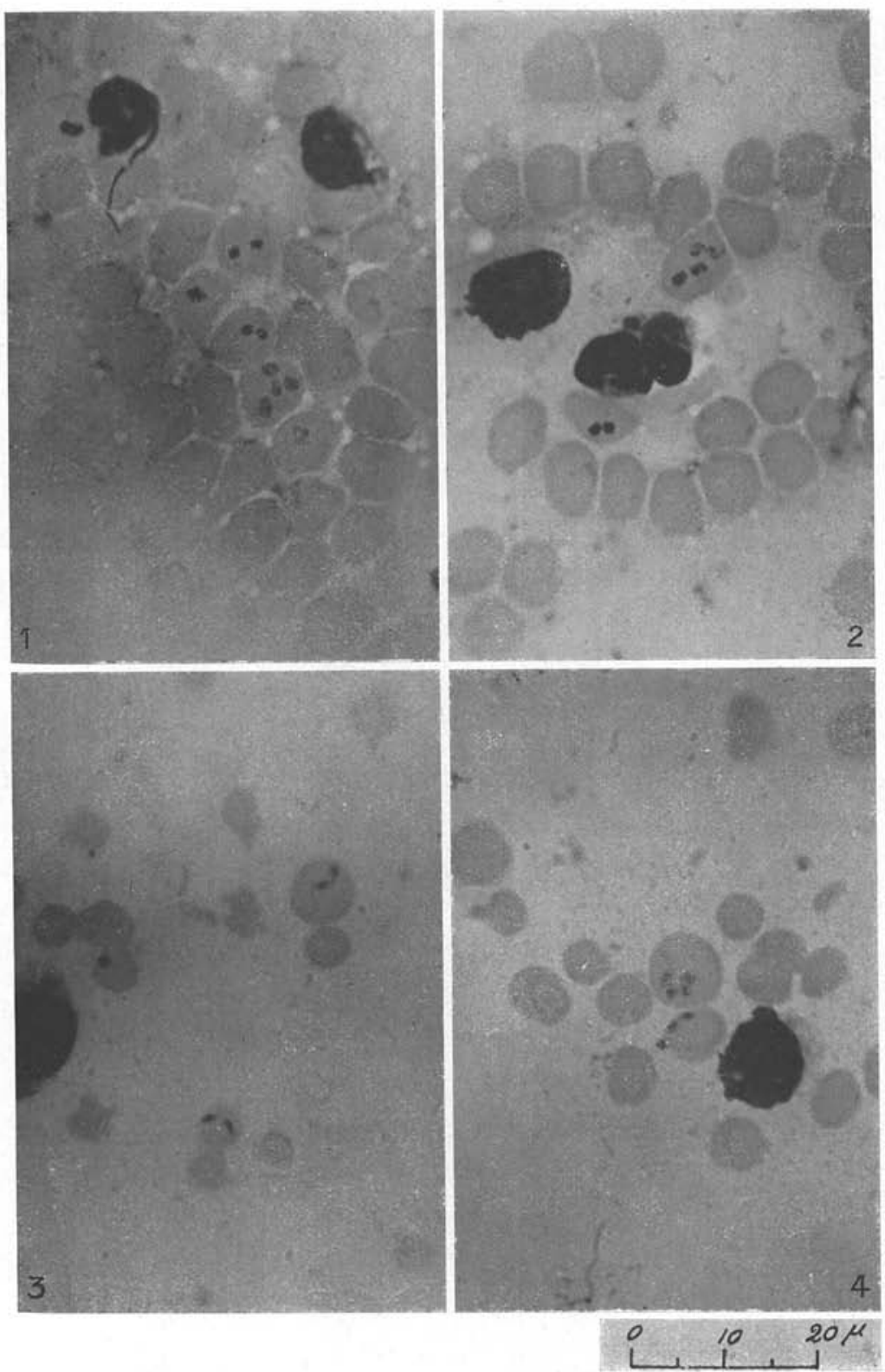

Masson et Cie, Editeuns 

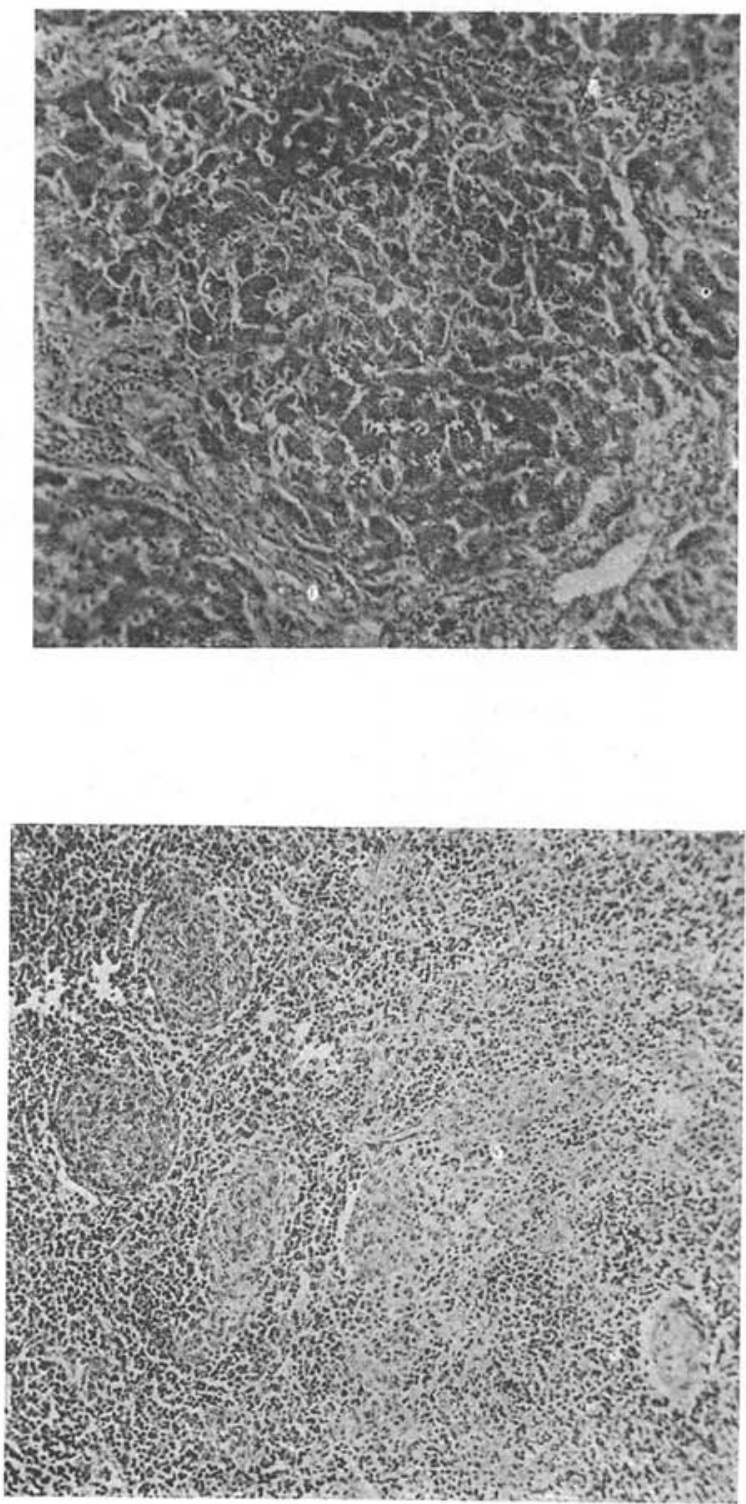

Masson et Cie, Editeuns 